\title{
Enhancement in lifetime of sensor node using Data Reduction Technique in Wireless Sensor Network
}

\author{
Nikhil Kumar Singh \\ Deptt.of ECE, Krishna Institute \\ of Engineering\& \\ TechnologyGhaziabad,UP,India
}

\author{
Ankit Kasana \\ Deptt. of ECE, Krishna Institute \\ of Engineering\& Technology \\ Ghaziabad,UP,India
}

\author{
Vibhav Kumar Sachan \\ Deptt. of ECE, Krishna Institute \\ of Engineering \& technology, \\ Ghaziabad, UP India
}

\begin{abstract}
In this paper data compression is becoming more popular, because it helps to reduce the size.A number of clustering protocols have been explored in order to obtain the effective energy usage in WSNs. It is based on randomized rotation of the $\mathrm{CHs}$ to distribute the energy load among the sensor nodes evenly in the entire network. Each node elects itself as a $\mathrm{CH}$ based on a probabilistic scheme and broadcasts its availability to all the sensor nodes present in the area. The received signal strength is the prime parameter for determining the communication distance between the nodes. The $\mathrm{CH}$ performs aggregation of the packets received from all the nodes present in their cluster. Also, all the nodes get a chance to become the $\mathrm{CH}$ to balance the overall energy consumption across the network. The aim ofClustering is one of the important methods for prolonging the network lifetime in wireless sensor networks (WSNs). It involves grouping of sensor nodes into clusters and electing cluster heads $(\mathrm{CHs})$ for all the clusters. CHs collect the data from respective cluster's nodes and forward the aggregated data to base station.by using Huffman encoding techniques [1].
\end{abstract}

\section{Keywords}

Wireless sensor network (WSN), cluster Head (CH), Sensor node, Huffman coding.

\section{INTRODUCTION}

A wireless sensor network (WSN) (here and there called a wireless sensor and on-screen character network (WSAN)) are spatially dispersed self-ruling sensors to screen physical or ecological conditions, for example, temperature, sound, weight, and so forth and to helpfully go their information through the network to a fundamental area. The more present day networks are bi-directional, additionally empowering control of sensor movement. The advancement of wireless sensor networks was persuaded by military applications, for example, combat zone reconnaissance; today such networks are utilized as a part of numerous mechanical and shopper applications, for example, modern procedure checking and control, machine wellbeing observing, etc.

The WSN is worked of "hubs" - from a couple to a few hundreds or even thousands, where every hub is associated with one (or once in a while a few) sensors. Each such sensor network hub has commonly a few sections: a radio handset with an interior reception apparatus or association with an outer receiving wire, a microcontroller, an electronic circuit for interfacing with the sensors and an energy source, for the most part a battery or an inserted type of energy reaping. A sensor hub may fluctuate in size from that of a shoebox down to the measure of a grain of dust, albeit working "bits" of bona fide infinitesimal measurements have yet to be made. The expense of sensor hubs is also variable, extending from a couple to several dollars, contingent upon the many-sided quality of the individual sensor hubs. Size and cost imperatives on sensor hubs result in comparing limitations on assets, for example energy, memory, computational velocity and correspondence transfer speed. The topology of the WSNs can fluctuate from a straight forward star network to a progressed multi-bounce wireless cross section network.The spread method between the bounces of the network can defeat or flooding. Group head choice in the WSNs is done utilizing certain conventions that are described further.WSN divides clusters each having a coordinator (cluster head) responsible for gathering the data from the nodes and sending it to the sink (base station). Sensors are often deployed densely to satisfy the coverage requirement, which enables certain nodes to enter the sleep mode thereby allowing significant energy savings. The cluster heads can be selected randomly or based on one or more criteria. Selection of cluster head largely affects WSNs lifetime. Ideal cluster head is the one which has the highest residual energy, the maximum number of neighbor nodes, and the smallest distance from base station. Simultaneous consideration of all these criteria in $\mathrm{CHs}$ selection is tedious task and can be solved using multiple attribute decision-making (MADM) approaches. A number of MADM approaches are reported and have been successfully applied in various scientific-, engineering-, and social-science based decision-making problems. These methods quantitatively select alternatives based on their multiple attributes/criteria. In real-time problem, it is often found that the estimation of the exact values of all the criteria is difficult. In such cases fuzzy-based MADM methodologies are found to be efficient and effective.

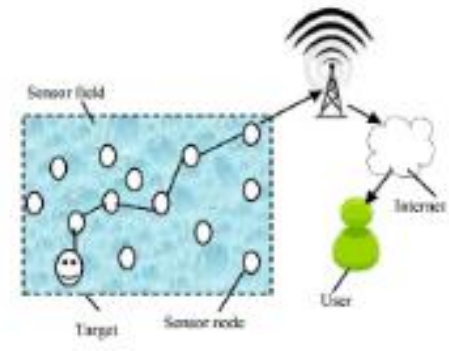

Fig 1: Architecture of wireless sensor network

\section{CLUSTERING HEAD}

Clustering is one of the important methods for prolonging the network lifetime in wireless sensor networks (WSNs). It involves grouping of sensor nodes into clusters and electing cluster heads $(\mathrm{CHs})$ [2] for all the clusters. $\mathrm{CHs}$ collect the data from respective cluster's nodes and forward the aggregated data to base station. A major challenge in WSNs is to select appropriate cluster heads. In this paper, we present a fuzzy decision-making approach for the selection of cluster 
heads. Fuzzy multiple attribute decision-making (MADM) approach is used to select $\mathrm{CHs}$ using three criteria including residual energy, number of neighbors, and the distance from the base station of the nodes.

\subsection{Clustering Formation}

Sensor nodes typically use irreplaceable power with the limited capacity, the node's capacity of computing, communicating, and storage is very limited, which requires WSN protocols need to conserve energy as the main objective of maximizing the network lifetime. An energy-efficient communication protocol LEACH, has been introduced [3] which employs a hierarchical clustering done based on information received by the BS. The BS periodically changes both the cluster membership and the cluster-head $(\mathrm{CH})$ to conserve energy.

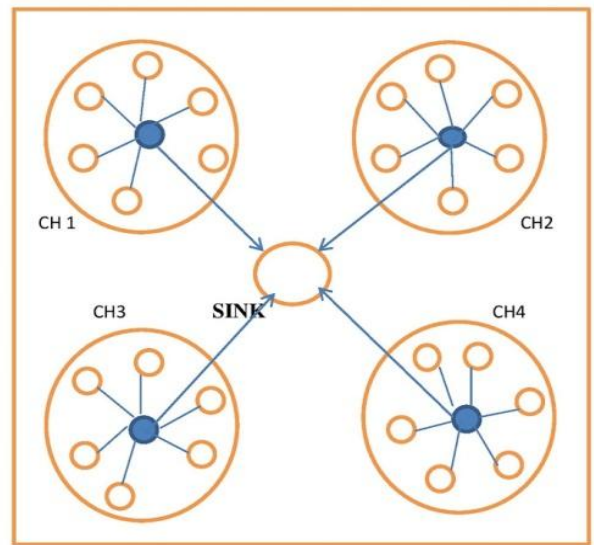

Fig 2: node, clustering node and role of sensor in typical WSN

The $\mathrm{CH}$ collects and aggregates information from sensors in its own cluster and passes on information to the BS. By rotating the cluster-head randomly, energy consumption is expected to be uniformly distributed. However, LEACH possibly chooses too many cluster heads at a time or randomly selects the cluster heads far away from the BS without considering nodes' residual energy. As a result, some cluster heads drain their energy early thus reducing the lifespan of WSN. In each round of the cluster formation, network needs to follow the two steps to select clusterhead and transfer the aggregated data. (i) Set-Up Phase, which is again subdivided in to Advertisement, Cluster Set-Up \& Schedule Creation phases. (ii) Steady-State Phase, which provides data transmission using Time Division Multiple Access (TDMA). The election of cluster head node in LEACH [3] has some deficiencies such as, Some very big clusters and very small clusters may exist in the network at the same time. Unreasonable cluster head selection while the nodes have different energy. Cluster member nodes deplete energy after cluster head was dead. The algorithm does not take into account the location of nodes, which may easily lead to cluster head node will rapidly fail. Motivated from this, so many clustering proposals are reported in the literature, suggesting different strategies of clusterhead selection and its role rotation.

\section{SYSTEM MODEL}

The following assumptions are considered in the present study.

(1)Nodes are dispersed randomly in a $100 * 100$ square unit region following a uniform distribution. (2)All the nodes send hello messages to the base station containing their local information. (3)The initial number of clusters is fixed by taking the optimum value and keeps on varying with the node density once the nodes start dying. The smaller clusters merge with the bigger ones. (4)The base station (BS) is a node with no energy constraint and enhanced computation capabilities and placed at the center of the field.(5) A simple radio energy dissipation model in transmitting a ' $\mathrm{k}$ ' bit message over a distance ' $d$ ' to achieve an acceptable signal-to-noise ratio (SNR)[4] is used. Energy consumption in data transmission can be estimated as

$E_{T x}=k * E_{\text {elec }}+k * \varepsilon_{f s} * d^{2}$

If $\mathrm{d} \leq d_{0}$

$E_{T x}=k * E_{\text {elec }}+k * \varepsilon_{\text {amp }} * d^{4}$

If $d \geq d_{0}$

Where $E_{\text {elec }}$ is the energy dissipated per bit to run the transmitter or the receiver circuit, $\varepsilon_{f s}$ is the energy consumed in the amplifier when $\mathrm{d} \leq d_{0}$ and $\varepsilon_{a m p}$ is the energy consumed in the amplifier when $d \geq d_{0}$ The energy consumed while reception is.

$E_{R x}=k * E_{\text {elec }}$

\section{RELATED WORK \\ 4.1 Energy Efficiency Design Of Wsn}

Energy efficiency is always one of the major goals in the design of WSN. Energy efficient protocols [4] have been explored for a long time. Previous work expects to achieve the goal of energy efficiency by designing energy efficient query protocols , routing protocols, such as energy efficient MAC protocols like energy efficient clustering and duty cycle management sensor network topology management and other energy efficient approaches However, these approaches mainly focus on finding some energy efficient path, designing better turn on/off schedules, forming energy efficient clusters, and so on, but none of them has examined the energy efficiency from the view of the data itself, i.e., to adapt the data sampling rate to the data dynamics and keep lazy when data consistency is maintained. Aggregation is one of the most common technologies used in wireless sensor network to save energy.

\subsection{Explanation Of Energy Protocols}

Wireless Sensor Networks (WSNs) consist of numerous sensors which send sensed data to base station. Energy conservation is an important issue for sensor nodes as they have limited power. In these schemes, cluster heads are selected for initial and subsequent data gathering rounds by evaluating an expression involving some probabilistic requirements, utilizing fixed parameters like number of cluster heads and round number. In LEACH, cluster head role is rotated among all sensor nodes by re-clustering the network after specific number of data gathering cycles called round. During each round, a fixed percentage of total network nodes are selected as cluster heads which then start cluster formation process by advertising their selection to the non-cluster head nodes that on receipt of these equal transmit power advertises, from different cluster heads, join one with highest received signal strength. Each node in the network chooses a random number between 0 and 1 and if this number is less than the evaluated adaptive threshold, selects itself as cluster head for 
the current round. In $\mathrm{LEACH}$, during some round, it is possible that none of the node selects itself as cluster head and all the nodes have to act as forced cluster heads. To improve upon, such a round is treated as cancelled in power efficient communication protocol and a fresh cluster head selection is carried out, independent of the current round. In resource adaptive schemes, information about the available node resources is utilized, while selecting cluster heads for the subsequent rounds. The scheme suggested in calculates the threshold considering residual energy, energy dissipated during current round and average node energy as additional parameters and makes the cluster head selection strategy energy adaptive. The nodes in the network take decision about their cluster head role carrying out a process similar to LEACH but with a resource adaptive threshold value. LEACH-B, Energy-LEACH and scheme in also adapt the LEACH threshold using different energy values. In HEED, sensor nodes use residual energy as a criterion to decide on their role as a cluster head and make up their mind setting the probability to a value expressed in terms of residual energy, maximum energy and the optimum percentage of cluster heads required for a particular data gathering round which is not allowed to fall below a minimum pre-defined threshold. In schemes, power optimized LEACH, ALEACH, EAMC , EAP, CEFCHS, FRCA, LEACH-M are suggests that node's remaining energy or residual energy as the main constrain to select a node as cluster head. In cluster based data gathering literature, some hybrid approaches are suggested combining clustering with, one or more of the, other architectures and increased energy efficiency is claimed. In M-LEACH that adjusting the nodes, Threshold function, when non clusterheads choose optimal cluster-head, they consider comprehensive nodes' residual energy and distance to basestation, then compare their performance, the simulation results show that the new strategy of cluster-heads election achieve great advance in sensor and in ACAER which periodically selects cluster nodes according to their coverage rate and residual energy. The EAMC can reduce the number of relays used for data transmission by minimizing the amount of the nodes in the root tree (that is cluster-head). Unequal Clusterbased Routing (UCR) protocol [2] groups the nodes into clusters of unequal sizes. Cluster heads closer to the base station have smaller cluster sizes than those farther from the base station, thus they can preserve some energy for the intercluster data forwarding and using decision tree algorithm to select the best node as a cluster head. Gradual Cluster head election Algorithm (GCA) which gradually elects cluster heads according to the proximity to neighbor nodes and the residual energy level and one-hop neighbor information (GCA-ON), which elects cluster heads based on Er and the relative location information of sensor nodes. LEACHimprove consider both energy and coverage together.

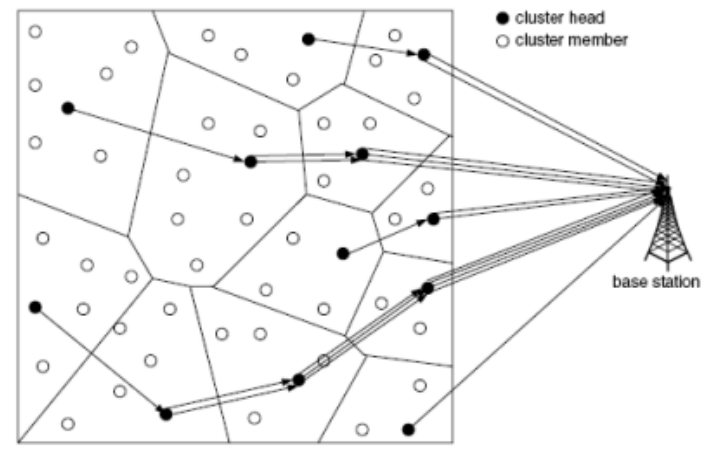

Fig 3: An overview of the UCR protocol.
In concentric clustering scheme, each node in the sensor network assigns itself a layer number, based on the received signal strength (RSSS) of the control message from the base station. The nodes with same layer numbers form a chain in their respective layers and one of these nodes is selected as a head node (cluster head) for that layer. The role of head node is rotated among the nodes in the same layer. LEACH protocol organizes the nodes by themselves. Regular nodes in cluster send data to Cluster Head $(\mathrm{CH})$. Cluster Head aggregates the data and sends to base Station. In LEACH Cluster Head Selection is based on the desired percentage of $\mathrm{CHs}$ for the network and number of times the node has been a $\mathrm{CH}$ so far. Each node should select a random number between the interval $0 \& 1$. If the generated random number is less than threshold then the node becomes a $\mathrm{CH}$ [6] for current round. Threshold is obtained by using the following formula:

$\mathrm{T}(\mathrm{n})=\{\mathrm{P} / 1-\mathrm{P} *(\mathrm{r} \bmod 1 / \mathrm{P})\}$

if $n \varepsilon \mathrm{G}$ Where, $P$ is the desired percentage of clusters; $r$ denotes the current round; $G$ denotes set of nodes that have not been $\mathrm{CHs}$ in the last 1/P rounds,

These assumptions are similar to those incorporated in and are as follow:

1. All the sensor nodes are homogeneous, stationary and energy constrained.

2. The base station is a high-energy node, located far away from the sensor nodes.

3. All the nodes can transmit with enough power to reach to the base station if needed.(iv) Nodes always have data to send to the end user and nodes located close to each other, have correlated data.

\subsection{Low Energy Adaptive Clustering Hierarchy (LEACH)}

Low Energy Adaptive Clustering Hierarchy (LEACH) [5] protocol has attracted intensive attention because of its energy efficient, simplicity and load balancing properties. LEACH is a cluster based protocol. The numbers of cluster heads and cluster members generated by LEACH are important parameters for achieving better performance. LEACH organizes nodes into clusters with one node from each cluster serving as a cluster-head $(\mathrm{CH})$. It randomly selects some predetermined number of nodes as cluster heads. $\mathrm{CHs}$ then advertise themselves and other nodes join one of those cluster heads whose signal they found strongest (i.e. the $\mathrm{CH}$ which is nearest to them). In this way a cluster is formed. The $\mathrm{CH}$ then makes a Time Divison Multiple Access (TDMA) schedule for the nodes under its cluster. The communication between different clusters is done through $\mathrm{CHs}$ in a Code Divison Multiple Access (CDMA) manner. The CHs collect the data from their clusters and aggregate it before sending it to the other $\mathrm{CHs}$ or base station (BS).

\subsection{Data-driven Approaches}

Data driven approaches is basically based on data reduction and energy-efficient data Acquisition. Data-driven approaches are planned to reduce the amount of sampled data by keeping the sensing accuracy within the acceptable level for application. Sampled data are generally having strong spatial and/or temporal correlation, so there is no need to communicate the redundant information to the sink. Energyefficient data acquisition schemes are mainly aimed at reducing the energy spent by the sensing subsystem. 


\section{HUFFMAN CODING}

The idea behind Huffman coding is to find a way to compress the storage of data using variable length codes. Our standard model of storing data uses fixed length codes. For example, each character in a text file is stored using 8 bits. There are certain advantages to this system. When reading a file, we know to ALWAYS read 8 bits at a time to read a single character. But as you might imagine, this coding scheme is inefficient. The reason for this is that some characters are more frequently used than other characters. Let's say that the character ' $\mathrm{e}$ ' is used 10 times more frequently than the character ' $q$ '. It would then be advantageous for us to use a 7 bit code for e and a 9 bit code for q instead because that could shorten our overall message length.

Huffman coding finds the optimal way to take advantage of varying character frequencies in a particular file. On average, using Huffman coding on standard files can shrink them anywhere from $10 \%$ to $30 \%$ depending to the character distribution. (The more skewed the distribution, the better Huffman coding will do.)

\section{PROBLEM FORMULATION}

Energy efficient protocols are the protocols that are designed for increasing the energy efficiency of the network. In these protocols a cluster head is selected for each group of nodes. The cluster head is selected for the communication of nodes with the sink. The network is divided into some areas and each area has its own cluster head. The nodes of that area do not directly communicate with the sink, they first send the data to the cluster head and the cluster head then sends the data to the sink. The problem with the earlier used energy efficient protocols were that the energy consumption was more that result in the reduction in the network's lifetime. Most the work is done on the reduction of the distance between the nodes so that the energy consumption is reduced. The security factor of the data that is transmitted is not taken in consideration. So there is a need of proposing a new approach in which the security of the data transmitted is also taken in the consideration. To make network more efficient the new protocol is to proposed.

\subsection{Simulation Result}

In this section, we discuss and compare simulation results of Data Reduction Technique used in WSNs. We used MATLAB as a simulation tool.

We deploy a random network of 100 nodes with initial energy equal to 0.5 joule in filed with dimensions of $100 \mathrm{~m} \times 100 \mathrm{~m}$.In simulated scenario sink placed at center of the network field.

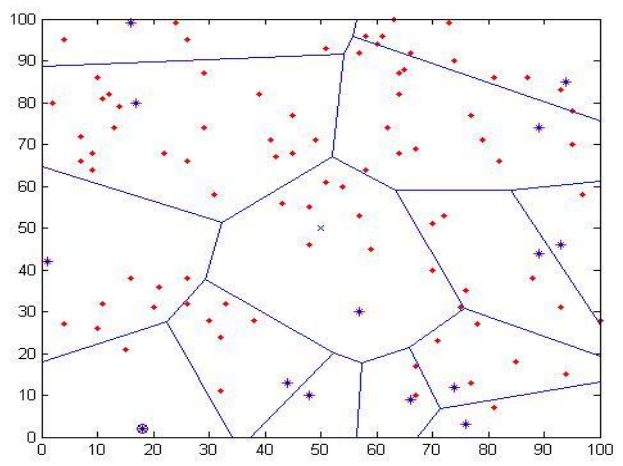

Fig 4: System model

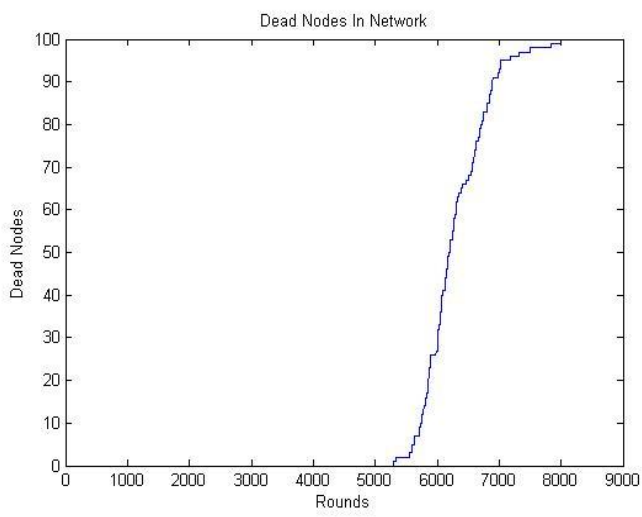

Fig 5: Number of Dead node

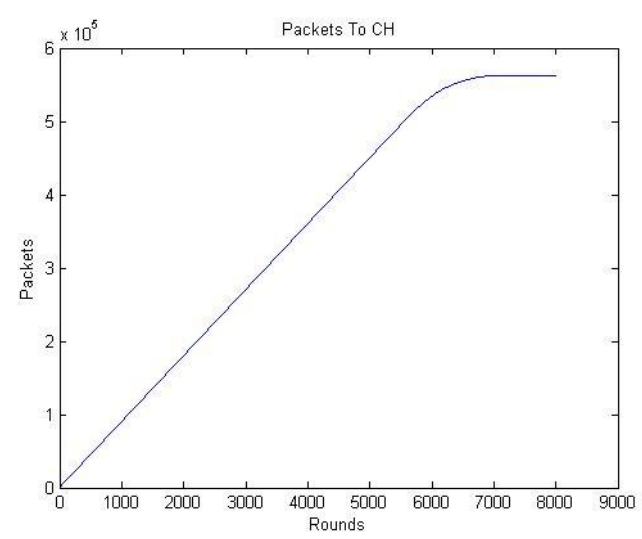

Fig 6: Number of Packet to CHs

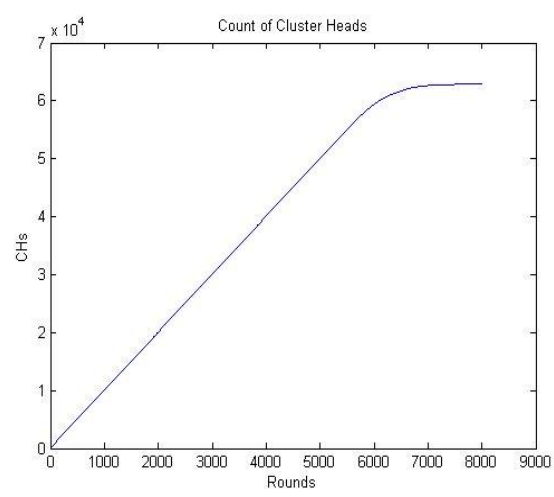

Fig 7: Count of CHs

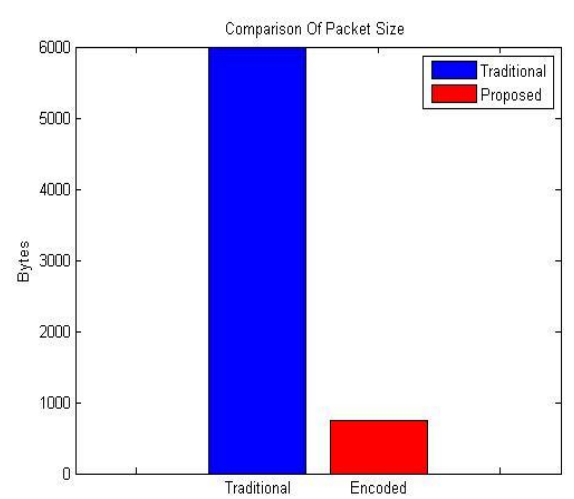

Fig 8: Packet size comparison 
We evaluated performance of our proposed strategy on the basis of certain parameter network stability period, network life time, and throughput.In this fig is clear that compare with alive node and round. And that network life time is enhanced significantly compare with other clustering approaches.

In our case the network remains Alive almost up to 8000 round assuring network life time to be more optimized. Moreover, it is also obvious that stability period is also improved i.e first node dies around 5400 rounds. Fig (5) represent unstable period of network when compared to other protocols it is clear that it also improved. Fig (7) represents $\mathrm{CHs}$ selected in every round for different clustering protocol.

\section{CONCLUSION}

In this paper, we studied various cluster head selection algorithm for data aggregation in wireless sensor network. But all have handle due to periodically select cluster head for this lots of energy is consumed. That's why we have proposed the most favorable algorithm for the efficient cluster head selection in which no need to select cluster head periodically, so lot of energy is saved in the wireless network. we studied arithmetic encoding methodology and Huffman Technique after than conclusion better than Huffman technique is data size reduce and packet size reduce. This is not only reduced the data size of the network but also reduce communication cost. The simulation result shows enhance in life time of sensor node.

\section{REFERENCES}

[1] Shrusti Porwal (2013), "Data Compression Methodologies for Lossless Data and Comparison between Algorithms" International Journal of Engineering Science and Innovative Technology (IJESIT)

[2] Ying Liang "Energy Adaptive Cluster-Head Selection for Wireless Sensor Networks" IEEE, December, 2005,

[3] Microsensor Networks", In Proc. 33rd HICS, 4-7 Jan, 20004-Anirooth Thonklin, W. Suntiamorntut "Load Balanced and Energy Efficient Cluster Head Election in Wireless Sensor Networks.

[4] Thein (29 January, 2010) "An Energy Efficient ClusterHead Selection for Wireless Sensor Networks

[5] Vibhav Kumar Sachan, Syed Akhtar Imam and M T Beg. Article: Energy-Efficient Communication Methods in Wireless Sensor Networks: A Critical Review. International Journal of Computer Applications 39(17):35-48, February 2012

[6] Himanshu Sharma, Vibhav Kumar Sachan and Syed Akhtar Imam. Article: Energy Efficiency of the IEEE 802.15.4 Standard in Wireless Sensor Networks: Modeling and Improvement Perspectives. International Journal of Computer Applications 58(9):12-19, November 2012
[7] Syed Akhtar Imam, Vibhav Kumar Sachan and Shivani Singh. Article: Data Aggregation based Cooperative MIMO System for Wireless Sensor Networks: Performance Analysis. International Journal of Computer Applications 90(4):1-7, March 2014.

[8] Syed Akhtar Imam, Vibhav Sachan and Shivani Singh Article: Performance Analysis of Different Diversity Schemes for Energy Efficient Wireless Sensor Network. IJCA Proceedings on 4th International IT Summit Confluence 2013

[9] Vibhav Kumar Sachan, Richa Maheshwari and Syed Akhtar Imam. Article: Energy Efficient Wireless Sensor Networks using Co-operative MIMO: A Technical Review. International Journal of Computer Applications 135(11):20-27, February 2016

[10] I. F. Akyildiz, W. Su, Y. Sankarasubramaniam, and E. Cayirci, "Wireless sensor networks: a survey," Computer Networks, vol. 38, no. 4, pp. 393-422, 2002.

[11] J. Yick, B. Mukherjee, and D. Ghosal, "Wireless sensor network survey,"Computer Networks, vol. 52, no. 12, pp. 2292-2330, 2008.

[12] C. F. Hsin and M. Liu, "Randomly duty-cycled wireless sensor networks:dynamic of coverage," IEEE Trans. Wireless Commun., vol. 5, no. 11,pp. 3182-3192, 2006

[13] Pratyay Kuila (2012) “An energy balanced distributed clustering and routing algorithm for Wireless Sensor Networks"

[14] W. Suntiamorntut "Load Balanced and Energy Efficient Cluster Head Election in Wireless Sensor Networks

[15] M. J. Handy "Low Energy Adaptive Clustering Hierarchy with Deterministic Cluster-Head Selection" IEEE, September, 2002.

\section{AUTHOR PROFILE}

Mr. Nikhil Kumar Singh has received his B.Tech degree in Electronic and Communication engineering from Prasad Institute of Technology (2010) affiliated to Gautam Buddh Technical Uneversity (GBTU) Lucknow, India and pursuing M.Tech in Electronics and Communication engineering from Krishna Institute of Engineering Technology Ghaziabad, affiliated to AKTU(Abdul Kalam Technical University), Uttar Pradesh Lucknow.His main Research areas of interest are enhancement in life time of sensor node using Huffman Coding e.t.c.

Mr. Ankit kasana has received his B.Tech in Electronic and Communication engineering from Collage \& Rural Technology (2011).affiliated to Gautam buddh Technical University (GBTU) lucknow,India and pursuing M.Tech in (ECE) from KIET Ghaziabad affiliated to AKTU U.P Lucknow.his main Research area enhancement in life time of sensor node by Energy efficient protocol. 